\title{
Suicide Inquiry in Primary Care: Creating Context, Inquiring, and Following Up
}

Steven D. Vannoy, $\mathrm{PbD}, \mathrm{MPH}$

Tonya Fancher, $M D^{2}$

Caitlyn Meltvedt, BA ${ }^{2}$

Jürgen Unützer, $M D, M P H, M A^{1}$

Paul Duberstein, $P b D^{3}$

Richard L. Kravitz, MD, MSPH ${ }^{2}$

'Department of Psychiatry and Behavioral Sciences, University of Washington, Seattle, Washington

${ }^{2}$ Center for Healthcare Policy and Research, and Department of Internal Medicine, University of California, Davis, Davis, California

${ }^{3}$ Center for the Study and Prevention of Suicide, Department of Psychiatry, University of Rochester Medical Center, Rochester, New York

\begin{abstract}
PURPOSE We wanted to describe the vocabulary and narrative context of primary care physicians' inquiries about suicide.

METHODS One hundred fifty-two primary care physicians (53\% to $61 \%$ of those approached) were randomly recruited from 4 sites in Northern California and Rochester, New York, to participate in a study assessing the effect of a patient's request for antidepressant medication on a physician's prescribing behavior. Standardized patients portraying 2 conditions (carpal tunnel syndrome and major depression, or back pain and adjustment disorder with depressed mood) and 3 antidepressant request types (brand-specific, general, or none) made 298 unannounced visits to these physicians between May 2003 and May 2004. Standardized patients were instructed to deny suicidality if the physician asked. We identified the subset of transcripts that contained a distinct suicide inquiry $(n=91)$ for inductive analysis and review. Our qualitative analysis focused on elucidating the narrative context in which inquiries are made, how physicians construct their inquiries, and how they respond to a patient's denial of suicidality.
\end{abstract}

RESULTS Most suicide inquiries used clear terminology related to self-harm, suicide, or killing oneself. Three types of inquiry were identified: (1) straightforward (eg, "Are you feeling like hurting yourself?"); (2) supportive framing (eg, "Sometimes depression gets so bad that people feel that life is no longer worth living. Have you felt this way?"); and (3) no problem preferred (eg, "You're not feeling suicidal, are you?"). Four inquiries were glaringly awkward, potentially inhibiting a patient's disclosure. Most (79\%) suicide inquiries were preceded by statements focusing on psychosocial concerns, and most (86\%) physician responses to a standardized patient's denial of ideation were followed up with relevant statements (eg, "I hope you would tell me if you did.").

CONCLUSION Although most suicide inquiries by primary care physicians are sensitive, clear, and supportive, some language is used that may inhibit suicide disclosure. Some physician responses may unintentionally reinforce patients for remaining silent about their risk. This study will inform future research in the development of quality improvement interventions to support primary care physicians in making clear, appropriate, and sensitive inquires about suicide.

Ann Fam Med 2010;8:33-39. doi:10.1370/afm.1036.

\section{INTRODUCTION}

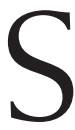
uicide is a stigmatized behavior accounting for more than 30,000 deaths per year in the United States. ${ }^{1}$ Although depression is highly prevalent among people who die by suicide, ${ }^{2}$ relatively few have sought mental health specialty services in the weeks preceding their death. ${ }^{3-5}$ In contrast, nearly one-half of the people who die by suicide have seen a primary care physician within a month of death. ${ }^{4,6}$ From a public health perspective, primary care visits may represent an important opportunity for suicide prevention.

An important aspect of case identification for suicide risk is the frequency with which physicians make suicide inquiries. Using data from 
the same parent study that supports this report, ${ }^{7}$ Feldman et $a 1^{8}$ reported an inquiry rate of $36 \%$ of primary care encounters in which female standardized patients portrayed either major depression or adjustment disorder with depressed mood. Suicide inquiry was more likely when standardized patients complained of major depression (vs adjustment disorder with depressed mood), and inquired about antidepressant treatment. Physician characteristics positively associated with addressing suicide included having a personal or vicarious experience with depression, asking more depression-related questions, and working in academic medical settings. Physicians' age, sex, self-reported confidence in treating depression, and practice size (solo vs group practice) were not associated with suicide inquiry. Nor were visit length or standardized patients' independent ratings of the primary care physician's patient-centered communication., 90

Not all questions are constructed equally, and none are presented in a vacuum. The primary goal of the medical interview is to elicit patient health problems, establish a diagnosis, generate a treatment plan, and secure the physician-patient relationship. ${ }^{11,12}$ Physicians are trained to use a question-and-answer format that promotes disclosure of problems while remaining efficient. Patients are socialized into a style of discourse with their physician in which they explain symptoms (often somatic) in exchange for an explanation of, and remedy for, their discomfort. First-encounter interviews prototypically start with problem-eliciting questions (eg, "What brings you in today?"). The normative presumption is that the patient has come seeking help for a problem and will openly disclose the nature of that problem upon questioning. Detecting and assessing risk for suicide relies on patient disclosure of factors related to potential self-harm, particularly suicidal ideation. ${ }^{13}$ Few patients spontaneously disclose suicidal thoughts, however, ${ }^{14,15}$ and few primary care physicians ask about suicide ideation. ${ }^{8,14}$

Suicide risk assessment challenges normative patient-physician communication by forcing the physician to probe for an often unstated concern. The way a question is worded can have consequences, such as communicating a physician's preference for a no-problem answer, ${ }^{16}$ which may be particularly salient when asking about stigmatized issues. Hence, we sought a deeper understanding of the process of suicide inquiry when it did occur within these encounters. In this article, we address the following questions regarding how physicians inquire about suicide: (1) in what narrative

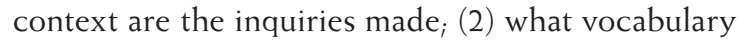
and sentence structure do physicians use when inquiring about suicide; and (3) how do physicians respond when a patient denies suicidal behavior? We use the term suicidal behavior to refer to suicide ideation (passive or active), preparation for a suicide (accumulating means, saying good-byes), and self-injurious behavior with intent to inflict a fatal wound.

\section{METHODS}

\section{Study Sample}

We performed a secondary analysis of data from a randomized controlled trial examining the effects of patient requests for antidepressant medications. Details of the original study have been reported elsewhere. ${ }^{7}$ In brief, standardized patients visited participating physicians' offices in unannounced visits. The standardized patients were white women in their 40s. Encounters were randomized to 2 different medical conditions and 3 different medication request types. Specifically for the medical condition, the standardized patients portrayed either carpal tunnel syndrome (purpose for visit) with major depression or low back pain (purpose of visit) and adjustment disorder with depressed mood. Medication requests were either associated with a specific antidepressant, a generic request for an antidepressant, or no request at all.

Board-certified and board-eligible family physicians and internists were recruited by mail with telephone follow-up from 4 physician networks: the University of California, Davis, Primary Care Network $_{i}$ Kaiser-Permanente in Sacramento, California; and Brown \& Toland Medical Group in San Francisco, California; and Excellus BlueCross BlueShield in Rochester, New York. Informed consent was obtained from the participating physicians. The institutional review boards at each participating institution approved the study protocol. ${ }^{7}$ Cooperation rates ranged from $53 \%$

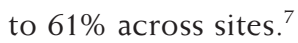

All standardized patient visits were conducted between May 2003 and May 2004 and were audio recorded using minidisc recorders concealed in the patients' handbags. ${ }^{9}$ Standardized patients made only 1 new-patient visit to each physician, and they were instructed to deny any experience of suicidal behaviors. As soon as possible after each visit, the standardized patients completed a form that included a question asking whether the physician asked about suicide. The standardized patient was instructed to answer yes to this item if she had been asked about suicide behavior by the physician, or if she was asked to complete a questionnaire that included an item about suicidal behavior. Reliability of reporting physician behavior during the encounter was high when compared with ratings by independent judges using visit audio recordings $(\kappa=0.82){ }^{7}$ 


\section{Data}

We analyzed transcripts from encounters in which standardized patients reported having been asked about thoughts of wanting to be dead, engaging in self-harm, or dying by suicide (108 of 298 encounters). ${ }^{8}$ The suicide inquiry was identified by a textbased search using the following keywords: suicid*, death, dying, kill*, hurt*, harm*, and disappear. (An asterisk indicates use of a wild card search in that any text string with the characters preceding the asterisk would match; for example, suicid* matches suicide or suicidal.) Transcripts that remained without an identified inquiry were reviewed in their entirety. Our data consisted of transcriptions of the audio-recorded encounters in electronic text format. The transcripts were organized in sequential verbal blocks, consisting of 1 or more contiguous uninterrupted statements attributed to either the patient or physician. A verbal block could be as short as a single word (eg, okay, yes) or as long as several paragraphs of continuous speech by 1 person. Our smallest unit of analysis was the verbal block. ${ }^{17}$

\section{Coding the Suicide Discourse}

Coding evolved through an iterative manner of reading transcripts and discussing impressions among the authors, who have expertise in psychology, internal medicine, suicidology, and psychiatry. Through inductive review we focused on 3 stages in the suicideinquiry process: context, inquiry, and response. Context refers to the topic of discussion at the time inquiry is made. We coded the current topic as in or out of context based on the whether the current topic related to psychosocial functioning or prototypical depression symptoms (eg, appetite). We defined inquiry as the statement that contained the suicide question. Response refers to the physician statements immediately subsequent to the standardized patient's denial of suicidal behavior. Three authors (T.F., C.M., S.D.V.) reviewed the inquiries and collaboratively identified categories based on both wording and sentence structure. Differences in interpretation were discussed until consensus was reached.

We began our analysis using a microanalytic approach $^{11}$ to identify the range and frequencies of linguistic variations in suicide inquiry. Specifically, we cataloged the word(s) used to refer to suicide, as well as the structure of the question itself. Physicians often frame inquiries into stigmatized topics in an attempt to minimize discomfort. ${ }^{18}$ Framing is achieved by joining the actual inquiry with a related clause that serves to normalize the question (eg, "Has this stress gotten to the point where you've had thoughts about killing yourself or anything like that?" or "Sometimes when people are feeling down, you know, they feel like they want to stop living or harm themselves, have you had thoughts like that?").

Restricting our analysis to the physician's statement containing the suicide inquiry left us feeling we were missing contextual factors that could strongly influence how the patient perceived the question. It is common in primary care discourse for physicians to jump from topic to topic, ${ }^{19}$ and we wanted to know the context in which these inquiries occurred. Two metaphors are useful here: Did the inquiries appear out of the blue? Or were they seamlessly woven into the interview? Furthermore, recognizing a unique nature of this data set in that all standardized patients denied suicidal behavior, we were interested in how physicians responded to the denial and how they transitioned to new topics.

We coded the 3 verbal blocks uttered by the physician preceding the inquiry as either in or out of context based on whether the topic being discussed was psychosocial. In the course of several iterations of comparing and discussing our coding, we agreed on a liberal definition for "in context" as any statement directly related to depression or more generally related to psychosocial function. For example, a statement such as, "Have you been able to sleep?" was coded as in context because it would likely appear in a dialogue assessing psychological status. As with statements preceding the inquiry, we coded the 3 verbal blocks uttered by the physician after the standardized patient's denial of suicidal behavior (response) as either in or out of context.

We report frequency counts for each of the categories identified to illustrate the variability in discourse.

\section{RESULTS}

Eighteen standardized patients made 298 visits to 152 physicians. The encounters analyzed here occurred in Sacramento, California $(\mathrm{n}=33)$, San Francisco, California $(\mathrm{n}=40)$, and Rochester, New York $(\mathrm{n}=35)$. Seventy-three visits (67\%) were to general internists, and 35 (33\%) were to family physicians; 74 visits (68\%) were to male physicians, and 34 visits (32\%) were to female physicians. ${ }^{7}$ Of the 108 encounters in which standardized patients reported being asked about suicide, 102 transcripts were available for qualitative analysis (6 were excluded because of inaudible recordings). Text-based searching for keywords found 85 transcripts containing clear physician suicide inquiries. The remaining 17 transcripts were read in their entirety. These manual searches identified suicide inquiries in 6 encounters. Ten of the remaining 11 transcripts contained no discussion of suicide, suggest- 
ing that the standardized patient had been administered a questionnaire with a suicide-related question that was not discussed in the encounter. One transcript was incomplete, leaving 91 complete transcripts with suicide dialogue. A sample verbal block illustrating units of analysis is provided in Appendix 1.

\section{Inquiry}

Our initial focus was on the linguistic structure of the inquiry itself. We identified the following 3 categories of linguistic content: direct inquiries that used a variation on the word suicide or killing (eg, "Are you feeling suicidal at all?" or "Has this stress gotten to the point where you've thought about killing yourself, or anything like that?"); indirect inquiries related to self-harm (eg, "Have you had thoughts of wanting to hurt yourself at all?" or "Do you worry about hurting yourself?"); and indirect inquiries related to hopelessness or passive death ideation (eg, "Do you have any feeling that life is not worth living?" or "Have you ever thought about death a lot?"). Inquiries using multiple terms were also present (eg, "... any thoughts of harming yourself, suicidal thoughts, or anything like that?"). The most common wording was related to self-harm (56\%), but almost as common were direct inquiries (48\%). Much less common $(13 \%)$ were indirect references to hopelessness or passive ideation (13\%). Physicians rarely asked question using a variant of the word dead (3\%).

Framed questions occurred in $24 \%$ of inquiries. No-problem ${ }^{16}$ inquiries use wording communicating that the physician either assumes, or prefers, a bestcase scenario. We coded no-problem preferences in 2 ways. The first were inquiries that used explicit negation, eg, "No thoughts of harming yourself, right?" $(20 \%, \mathrm{n}=19)$. The second, a more subtle form of negative polarity, is the use of the words any, anything, or ever in the question, which has been shown to influence the frequency of patient disclosure ${ }^{20}$ and was common in this data set, eg, "Have you had any thoughts of suicide?" $(9 \%, \mathrm{n}=44)$.

Although the overwhelming majority of inquiries were easily identifiable as questions about suicide, several particularly vague inquiries led the confused standardized patient to request clarification, (eg, "Do you mean suicide?") Appendix 2 depicts a stark example of a perplexing exchange replete with confusing terminology and an out-of-context follow-up statement.

\section{Context of Suicide Inquiry}

Physician-patient communication in primary care encounters often jumps from topic to topic and covers numerous patient complaints. ${ }^{19}$ Most (85\%) of the preceding blocks were coded as being in context. Seventy-nine percent of inquiries were preceded by 3 consecutive in-context blocks. In a small, but nonnegligible, proportion of encounters (10), there was no incontext physician statement in the 3 preceding blocks.

\section{Response}

All standardized patients were instructed to deny suicidal thoughts when asked by the physician if they were suicidal. In this section, we examine how physicians' responded to patients' denials. In discussing the follow-up blocks, we were struck by 2 distinct variations within the contextual responses. Some statements seemed to be clearly intended to open the door for disclosure (eg, "If anything changes, please call.") or to seek reassurance (eg, "Are you sure?"). These supportive follow-up statements were common $(37 \%, \mathrm{n}=34)$. Others $(26 \%, \mathrm{n}=24)$ more likely served to deter discussion or even close off future discussion of the topic (eg, "Okay, good. I didn't think so.") by reinforcing the no-problem response. We chose not to code isolated one-word responses, such as right, good, or okay, as positive or negative. These responses are addressed in the discussion section below.

As with preinquiry dialogue, most follow-up statements were coded as being in context (88\%). Likewise, most physicians (79\%) kept the discourse in context for all 3 follow-up statements, whereas a complete absence of in-context follow-up statements was rare (2\%).

\section{DISCUSSION}

In this detailed qualitative analysis of 91 primary care visits that were audio recorded, most inquiries were sensitive and straightforward. Generally, physicians seamlessly wove the topic of suicide into the conversational fabric that was focused on psychosocial functioning. Unlike many other medical topics, suicide inquiries are embedded within a contextual discourse in which depression and psychosocial functioning are clearly appropriate for discussion. The vocabulary used was overwhelmingly clear with respect to suicide being the target. It was rare for the standardized patient to ask physicians to clarify or repeat their questions. Perhaps the most encouraging finding in this analysis was that physicians rarely responded to the standardized patient's denial of suicide ideation by jumping to a fresh topic. Many responded by probing for more information or by expressing supportive statements aimed at reassuring that the physician is concerned for their safety.

A sizable portion of physicians framed their inquiry, which may have the effect of destigmatizing and emphasizing the importance of the question. Framing is considered to be beneficial when discussing stigmatized topics with patients and promoted in contem- 
porary physician education programs on preventative interventions. ${ }^{21}$

Some aspects of a physician's discourse may inhibit patient disclosure. A small portion of inquiries were not prefaced by in-context statements. Failing to create a context before the inquiry may catch patients off guard and inhibit subsequent disclosure. We were also struck by the use of no-problems-expected phrasing, which may result in a subtle but distinct message to the patient that denying suicide ideation is the correct response. This type of phrasing (eg, "You're not feeling suicidal are you?") is problematic for 2 reasons. First, closed questions may inhibit patient disclosure. ${ }^{16}$ Second, questions worded in a polarized fashion may lead the patient toward a confirming, or no-problem, response. ${ }^{16,22,23}$ This question format has been identified as specifically problematic for patient-physician communication related to other stigmatized health concerns. ${ }^{24}$

Likewise, there were responses to patient denials that raised concerns that the physicians were reinforcing the patient for denying suicide behavior. We found it common for physicians to interpose exchanges with brief utterances, such as "okay," "good," or "right." Although more subtle than the former example, the consensus of our raters was consistent with the literature in that these utterances close the current topic and reinforce the no-problem status. ${ }^{25}$ Similarly, failure to follow-up patient responses with in-context statements may communicate lack of interest (or discomfort) with the topic and therefore inhibit future reporting. Without knowing the impact of such communication on patients' willingness to disclose, these concerns must be considered as questions for future study.

Finally, although some physicians pursued additional information in their follow-up dialogue, we noted the absence of any questioning regarding access to firearms, stockpiling medications, or other lethal means.

\section{Limitations}

We lacked the data necessary to determine motivations behind the primary care physicians' behaviors we observed or the subjective impact the observed behaviors might have on real patients. Hence, our concerns about question format, sequencing, and response style were not confirmed by subjective or objective reports from authentic encounters.

The use of standardized patients limits the ability to generalize these findings beyond first-visit physician encounters with middle-aged white women. Although there is evidence of differences between initial and return primary care visits, ${ }^{26}$ our focus on initial visits of patients with depression symptoms represents an important opportunity to identify and respond to suicide risk. Further studies are necessary to determine what impact physician-patient familiarity might have on suicide communication. Using only middle-aged white women as standardized patients also raises concerns of how physician behavior might vary for other patient groups. The sex of the patient plays a smaller role in shaping medical encounters than does the sex of the physician. ${ }^{27}$ Specifically, differences in behavior related to psychiatric conditions do not appear to be influenced by the patient's sex. ${ }^{28,29}$ Based on differences in mental health service utilization, racial background is also an important factor that may influence how physicians address suicide risk. ${ }^{30,31}$ Likewise, age is particularly important, given the suicide rates in older men and documented bias in addressing this risk in primary care. ${ }^{32}$ Using standardized patients also limits our ability to understand the impact physician communication has on the willingness of real patients with actual suicidal behavior to disclose. The standardized patients were instructed not to spontaneously volunteer the presence or absence of suicide behavior, and to deny the presence of suicide behavior if asked. Finally, the participation rates of our primary care physicians ranged from $53 \%$ to $61 \%$ and may possibly reflect a participation bias that favored physicians who were more confident in their communication styles to participating in such a study. Consequently, these findings may represent a best-case scenario.

Previous studies have shown that there is significant physician variability in inquiry about suicidal behavior. ${ }^{8,14}$ Despite the low frequency of physician inquiries regarding suicidal behavior, we found that most inquiries were sensitive, appropriate, and supportive. By presenting a descriptive framework for how suicide is discussed when the topic is broached, we identified several potential challenges in promoting patient disclosure, which require further study. Future experimental research should determine the impact of naturalistic variations in inquiry style with ensuing patient help-seeking behavior.

Our descriptive framework may be useful in developing educational interventions to assist physicians who are inhibited from asking about suicidal behavior. Such interventions would emphasize generating a psychosocial context, using inquiries embedded in a supportive framework, and following up all patient responses with comments that assure the patient that the physician is open to discussing this topic in the future, if appropriate. Practicing physicians may benefit from seeing how their peers approach suicide inquiry when developing their own linguistic style for addressing this topic. Training for primary care physicians should include more practice on holding discussions related to suicide. Such training should raise awareness of methods for creating a safe environment for patients 


\section{Appendix 1. Example Areas of Analytic Focus Within 1 Verbal Block}

\section{Context}

Doctor: There been some life changes recently with this? [in context psychosocial]

Standardized patient: No, there hasn't been a thing.

Doctor: Okay. Are you able to get out of bed on the weekends or during the day; you find yourself wanting to get out of bed? [in context psychosocial/depression]

Standardized patient: Well I am tired; you know, I always get up out of bed.

Doctor: Okay. [in context by virtue of not being overtly out of context]

Standardized patient: Take a shower, get dressed, and all that.

\section{Inquiry}

Doctor: Have you had thoughts of wanting to hurt yourself at all? Or hurt anyone else?

Standardized patient: No.

\section{Response}

Doctor: Have you ever in the past ever, like when you were bummed out in your 20s? In the 20s, did you want to hurt yourself or try to hurt yourself? [in context pursuing additional information]

Standardized patient: No, I tried to get a job. Other than that I.... (voice fades)

Doctor: Okay. Have you thought about doing some counseling? [in context psychosocial/depression]

Standardized patient: Well, it's, it's entered my mind, uh, you know, I haven't really talked about it. You know, I thought, well, I don't know why, I don't know why this all started, I mean uh....

Doctor: Well, I think you have a couple of options here, I mean, it sounds like you probably are, you know, depressed. You know, one of the things; on your last physical did they do labs on you? I mean when you were seen 3 months ago, did they check your thyroid... [in context depression]

\section{Appendix 2. Example of Confusing Inquiry and Off-Topic Follow-Up}

Doctor: Have you felt like the bridge?

Standardized patient: The bridge?

Doctor: Have you felt like doing away with yourself?

Standardized patient: No.

Doctor: Good then. Well, let's check you over.

to disclose stigmatized behaviors, methods for specifically asking about suicide ideation, and how to both pursue corroborating information and communicate a supportive stance in light of potentially lethal psychological distress

To read or post commentaries in response to this article, see it online at http://www.annfammed.org/cgi/content/full/8/1/33.

Key words: Suicide, primary health care, depression

Submitted May 7, 2008; submitted, revised, May 19, 2009; accepted June 12,2009

A portion of the material in this article was presented as an oral presentation at the 2008 regional meeting of the California/Hawaii Society of
General Internal Medicine Regional Meeting, San Francisco, California, and the 2008 National Society of General Internal Medicine Regional Meeting, Pittsburgh, Pennsylvania.

Funding support: Original data collection was supported by a grant (R01 MH064683) from the National Institute of Mental Health. Data analysis and manuscript preparation for this manuscript was supported by National Institute of Mental Health grants T32MH73553 (Dr Vannoy), KL2RR025015 (Dr Vannoy), K24MH72756 (Dr Kravitz), K24MH072712 (Dr Duberstein), P50MH073511 (Dr Fancher).

Disclaimer: The design, conduct, data collection, analysis, and interpretation of the results of this study were performed independently of the funders. The funding agencies also played no role in review or approval of the manuscript.

Acknowledgments: The authors wish to thank Blue Shield of California, the UCD Primary Care Network, Western Health Advantage (Sacramento, Calif), Kaiser Permanente (Sacramento, Calif), Brown \& Toland IPA (San Francisco, Calif), and Excellus BlueCross BlueShield (Rochester, New York). We are also indebted to 18 standardized patients and to participating physicians and their office staff.

\section{References}

1. WISQARS. National Center for Injury Prevention and Control. WISQARS (Web-based Injury Statistics Query and Reporting System). 2007. http://www.cdc.gov/ncipc/. Accessed May 24, 2007.

2. Conwell Y, Duberstein PR, Cox C, Herrmann JH, Forbes NT, Caine ED. Relationships of age and axis I diagnoses in victims of completed suicide: a psychological autopsy study. Am J Psychiatry. 1996;153(8):1001-1008.

3. Mann JJ, Apter A, Bertolote J, et al. Suicide prevention strategies: a systematic review. JAMA. 2005294(16):2064-2074.

4. Luoma JB, Martin CE, Pearson JL. Contact with mental health and primary care providers before suicide: a review of the evidence. Am J Psychiatry. 2002;159(6):909-916.

5. Pirkis J, Burgess P. Suicide and recency of health care contacts. A systematic review. Br J Psychiatry. 1998;173:462-474.

6. Carney SS, Rich CL, Burke PA, Fowler RC. Suicide over 60: the San Diego study. J Am Geriatr Soc. 1994;42(2):174-180.

7. Kravitz RL, Epstein RM, Feldman MD, et al. Influence of patients' requests for direct-to-consumer advertised antidepressants: a randomized controlled trial. JAMA. 2005;293(16):1995-2002.

8. Feldman MD, Franks P, Duberstein PR, Vannoy S, Epstein R, Kravitz RL. Let's not talk about it: suicide inquiry in primary care. Ann Fam Med. 2007;5(5):412-418.

9. Geraghty EM, Franks P, Kravitz RL. Primary care visit length, quality, and satisfaction for standardized patients with depression. J Gen Intern Med. 2007;22(12):1641-1647.

10. Brown J, Stewart M, Ryan B. Assessing Communication Between Patients and Physicians: The Measure of Patient-Centred Communication (MPCC). 2001. Working Paper Series. 2nd ed. London, Ontario: Thames Valley Family Practice Research Unit and Centre for Studies in Family Medicine; 2001. Working Paper 952.

11. Heritage J, Maynard DW. Communication in Medical Care : Interaction Between Primary Care Physicians and Patients. Cambridge, UK; New York: Cambridge University Press; 2006.

12. Haidet P, Paterniti DA. "Building" a history rather than "taking" one: a perspective on information sharing during the medical interview. Arch Intern Med. 2003;163(10):1134-1140.

13. Raue PJ, Brown EL, Meyers BS, Schulberg HC, Bruce ML. Does every allusion to possible suicide require the same response? J Fam Pract. 2006;55(7):605-612. 
14. Nutting PA, Dickinson LM, Rubenstein LV, Keeley RD, Smith JL, Elliott CE. Improving detection of suicidal ideation among depressed patients in primary care. Ann Fam Med. 2005;3(6):529-536.

15. Verger P, Brabis PA, Kovess V, et al. Determinants of early identification of suicidal ideation in patients treated with antidepressants or anxiolytics in general practice: a multilevel analysis. J Affect Disord. 2007;99:253-257.

16. Boyd $\mathrm{E}$, Heritage J. Taking the history: questioning during comprehensive history taking. In: Communication in Medical Care: Interaction Between Primary Care Physicians and Patients. Cambridge: CUP; 2006:151-184.

17. Gee JP. An Introduction to Discourse Analysis: Theory and Method. London; New York: Routledge; 2005.

18. Gerbert B, Caspers N, Bronstone A, Moe J, Abercrombie P. A qualitative analysis of how physicians with expertise in domestic violence approach the identification of victims. Ann Intern Med. 1999;131(8):578-584

19. Tai-Seale M, McGuire TG, Zhang W. Time allocation in primary care office visits. Health Serv Res. 2007;42(5):1871-1894.

20. Heritage J, Robinson JD, Elliott MN, Beckett M, Wilkes M. Reducing patients' unmet concerns in primary care: the difference one word can make. J Gen Intern Med. 2007;22(10):1429-1433.

21. Rhodes KV, Frankel RM, Levinthal N, Prenoveau E, Bailey J, Levinson W. "You're not a victim of domestic violence, are you?" Provider patient communication about domestic violence. Ann Intern Med. 2007;147(9):620-627.

22. Horn LR. A Natural History of Negation. Chicago, IL: University of Chicago Press; 1989.

23. Pomerantz A. Offering a candidate answer: an information seeking strategy. Commun Monogr. 1988;55(4):360-373.
24. Rhodes KV, Frankel RM, Levinthal N, Prenoveau E, Bailey J, Levin son W. "You're not a victim of domestic violence, are you?" Provider patient communication about domestic violence. Ann Intern Med. 2007;147(9):620-627.

25. Beach WA. Preserving and constraining options: "Okays" and "official" priorities in medical interviews. In: Morris, GH, Cheneil RJ, eds. The Talk of the Clinic: Explorations in the Analysis of Medical and Therapeutic Discourse. Hillsdale, NJ: Lawrence Erlbaum Associates, Inc; $1995: 259-289$

26. Bertakis KD, Azari R, Callahan EJ, Robbins JA, Helms LJ. Comparison of primary care resident physicians' practice styles during initial and return patient visits. J Gen Intern Med. 1999;14(8):495-498.

27. Bertakis KD, Azari R. Patient gender and physician practice style. J Womens Health (Larchmt). 2007;16(6):859-868.

28. Hall JA, Roter DL. Patient gender and communication with physicians: results of a community-based study. Womens Health 1995;1(1):77-95.

29. Franks $P$, Bertakis KD. Physician gender, patient gender, and primary care. J Womens Health (Larchmt). 2003;12(1):73-80.

30. Snowden LR, Pingitore D. Frequency and scope of mental health service delivery to African Americans in primary care. Ment Health Serv Res. 2002;4(3):123-130.

31. Cooper LA, Gonzales JJ, Gallo JJ, et al. The acceptability of treatment for depression among African-American, Hispanic, and white primary care patients. Med Care. 2003;41(4):479-489.

32. Uncapher $H$, Areán PA. Physicians are less willing to treat suicidal ideation in older patients. J Am Geriatr Soc. 2000:48(2):188-192. 